\title{
THE ROLE OF SURFACE WAVES IN THE OCEAN MIXED LAYER
}

\author{
Fangli QIAO, Guohong FANG, Changshui XIA, Yongzeng YANG, Jian MA and Yeli Yuan \\ (First Institute of Oceanography, State Oceanic Administration, Qingdao, CHINA)
}

The ocean surface ML determines the lower atmospheric boundary conditions, and controls mass, momentum and energy fluxes across the air-sea interface. Incorrect parameterizations of the mixing processes essentially render the atmospheric and oceanic dynamics to be either decoupled or coupled incorrectly. Because the ocean covers three quarters of the global surface, it is essential that we correctly model the ocean ML, so that we can better model the climate. As the mixing process is essentially an energy balance problem, waves, as the most energetic motions at the ocean surface, should play a controlling role. Unfor-tunately, in most ocean dynamics studies, wave motions have always been treated separately from the ocean circulation6. Yet, it can be shown that even the second order wave-induced mass transport velocity, the Stokes drift, can produce a mass transport comparable to the wind-induced Ekman current7,8. Many recent rigorous studies9 have prompted a serious examination of the relevance of the micro-scale wave motions in the large-scale ocean dynamics.

In addition to mass transport, a far more important role of wave motions is its effects in the dynamics of the ML. Our study indicates that the wave-induced mixing penetration depth can reach nearly $100 \mathrm{~m}$ in high latitude and about $30 \mathrm{~m}$ in tropical areas5. Here, the parameterization of the wave effects has a long history ranging from considering the straining of vorticity by the irrotational waves 10 , which proved to be too feeble for any dynamic consequence, to the eventual realization of the importance of breaking waves 11, which proved to be highly relevant, but too difficult to implement. Numerous attempts notwithstanding, a proper solution is still wanting. Yet the role of waves is so obvious from a statistical consideration and observations 12-14 that it cannot be ignored for the large-scale ocean problems.

Previously, most ocean circulation models have overlooked the role of the surface waves. As a result, these models have produced insufficient vertical mixing, with an under-prediction of the mixing layer (ML) depth and an over-prediction of the sea surface temperature (SST), particularly during the summer season1-3. As the ocean surface layer determines the lower boundary conditions of the atmosphere, this deficiency has severely limited the performance of the coupled oceanatmospheric models and hence the climate studies. To overcome this shortcoming, we have established a new parameterization for the wave effects in the ML model4,5 that will correct this systematic error of insufficient mixing. The new scheme has enabled the mixing layer to deepen, the surface excessive heating to be corrected, and an excellent agreement with observed global climatologic data. Our study indicates that the surface waves are essential for ML formation, and that they are the primer drivers of the upper ocean dynamics; therefore, they are critical for climate studies. 